\title{
Anti-cytotoxic T lymphocyte antigen-4 antibodies in melanoma
}

\author{
Giulio Tosti \\ Emilia Cocorocchio \\ Elisabetta Pennacchioli \\ Divisione Melanomi e Sarcomi, \\ Istituto Europeo di Oncologia, \\ Milano, Italy
}

This article was published in the following Dove Press journal:

Clinical, Cosmetic and Investigational Dermatology

16 October 2013

Number of times this article has been viewed
Correspondence: Elisabetta Pennacchioli Istituto Europeo di Oncologia, via Ripamonti 435, 20I4I, Milan, Italy Email elisabetta.pennacchioli@ieo.it

\begin{abstract}
Approaches aimed at enhancement of the tumor specific response have provided proof for the rationale of immunotherapy in cancer, both in animal models and in humans. Ipilimumab, an anti-cytotoxic T lymphocyte antigen-4 (CTLA-4) antibody, is a new generation immunotherapeutic agent that has shown activity in terms of disease free and overall survival in metastatic melanoma patients. Its use was approved by the US Food and Drug Administration in March 2011 to treat patients with late stage melanoma that has spread or that cannot be removed by surgery. The mechanism of action of CTLA-4 antibodies in the activation of an antitumor immune response and selected clinical studies of ipilimumab in advanced melanoma patients are discussed. Ipilimumab treatment has been associated with immune related adverse events due to T-cell activation and proliferation. Most of these serious adverse effects are associated with the gastrointestinal tract and include severe diarrhea and colitis. The relationship between immune related adverse events and antitumor activity associated with ipilimumab was explored in clinical studies. Potential biomarkers predictive for clinical response and survival in patients treated with anti-CTLA-4 therapy are presently under investigation. Besides the conventional patterns of response and stable disease as defined by standard Response Evaluation Criteria in Solid Tumors criteria, in subsets of patients, ipilimumab has shown patterns of delayed clinical activity which were associated with an improved overall survival. For this reason a new set of response criteria for tumor immunotherapy has been proposed, which was termed immune related response criteria. These new criteria are presently used to better analyze clinical activity of immunotherapeutic regimens. Ipilimumab is currently under investigation in combination with other treatments, such as chemotherapy, target agents, radiotherapy, and other immunotherapeutic regimens.
\end{abstract}

Keywords: CTLA-4, CTLA-4 blockade, ipilimumab, metastatic melanoma, immunotherapy

\section{Introduction}

Approaches aimed at enhancement of the tumor specific response have provided proof for the rationale of immunotherapy in cancer, both in animal models and in humans. However, while many immunotherapeutic strategies generated potent and specific antitumor activity based on laboratory tests, the achievement of clinically meaningful and prolonged objective responses in cancer patients has been rarely observed. One reason for these results may be that many molecules identified as therapeutic targets in human cancer are self or "self altered" antigens, which may be either aberrantly expressed or overexpressed on malignant cells. Overcoming peripheral tolerance to these tumor associated targets may be of fundamental importance for the success of immune based therapies. ${ }^{1}$ 
Antibodies that are able to target specific antigens on the surface of cancer cells have emerged as challenging approaches in modern oncology. Trastuzumab, a recombinant humanized monoclonal antibody directed against the extracellular domain IV of HER2 (human epidermal growth factor receptor, type 2) in breast cancer, and rituximab, which binds CD20 on lymphoid cells, have become a mainstay in the therapy of HER2 overexpressing breast cancer and, respectively, in a variety of B-cell malignancies. ${ }^{2} \mathrm{~A}$ challenging strategy of using antibodies to stimulate the immune response was pioneered in the mid 1990s when the role of cytotoxic T lymphocyte antigen-4 (CTLA-4) as an inhibitory signal for the T-cell response became clearer. ${ }^{3}$

\section{The role of CTLA-4}

The T-cell compartment of adaptive immunity is regulated at multiple levels to prevent inappropriate activation (ie, autoimmunity) and the inhibitory activity exerted by CTLA-4 represents a crucial checkpoint at the periphery. The role of CTLA-4 in the regulation of the T-cell response became evident after the generation of specific monoclonal antibodies.

Anti-CTLA-4 antibodies enhanced proliferation of T-cells activated via anti-CD3 and anti-CD28. ${ }^{4}$ T-cell proliferation was partially inhibited by low levels of B7-2 on freshly explanted T-cells and this inhibition was obtained via antiCTLA-4, and further, crosslinking of CTLA-4 together with the T-cell receptor (TCR) and CD28 strongly inhibited proliferation and interleukin (IL)-2 production. ${ }^{5}$ Together with the finding that anti-B7 antibodies enhanced responses of T-cells activated by anti-CD3 along with anti-CD28 antibodies, these data suggested that CTLA-4 blockade resulted in removal of inhibitory signals. ${ }^{6,7}$ In vitro, mouse splenocytes stimulated with Staphylococcal enterotoxin B resulted in inhibition of T-cell responses in the presence of antibodies against B7 or Fab fragments of anti-CD28 antibodies and, conversely, in increased T-cell responses in the presence of Fab fragments of anti-CTLA-4 antibodies. Furthermore, blocking anti-B7 antibodies along with anti-CD28 antibodies augmented the response. ${ }^{8}$ Finally, the observation that anti-CD28 antibodies inhibited, whereas anti-CTLA-4 antibodies enhanced T-cell responses in vivo, supported the hypothesis that CD28 and CTLA-4 exert opposing effects upon T-cell activation., ${ }^{3,8}$

Definitive evidence of the role of CTLA-4 in the inhibition of T-cell activation and proliferation came from knockout mice. CTLA-4 deficient mice developed a fatal lymphoproliferative disorder consisting of a CD28 dependent expansion of autoreactive T-cells in lymph nodes, spleen, and nonlymphoid tissues. ${ }^{9}$ T-cell activation started 5 to 6 days after birth and the mice died at the age of 3-4 weeks due to lymphocytic infiltration of nonlymphoid tissues and autoimmunity. ${ }^{10-12}$

\section{Mechanism of action of CTLA-4}

CD4+ and CD8+ T-cells are activated by at least two signals between T-cells and antigen presenting cells (APCs). The first signal consists in the presentation of an antigen to TCR by a major histocompatibility complex molecule on an APC. The second costimulatory signal is generated by binding of the CD28 receptor on T-cells to B7 molecules on APCs. ${ }^{13}$ The activated $\mathrm{CD} 28$ receptor engages the same $\mathrm{B} 7$ molecules as the inhibitory CTLA-4 receptor, although with a reduced affinity (from 500 to 2,500 fold). CD28 and CTLA-4 display a different pattern of expression on T-cells: while CD28 is constitutively expressed on the surface of T-cells, CTLA-4 is slightly detectable in conventional naïve T-cells and appears upon T-cell activation. ${ }^{3,14}$

CTLA-4 binding to B7 molecules negatively regulates activated T-cells through different mechanisms. In addition to this competition with CD28, CTLA-4 can directly inhibit TCR signals, reduce IL-2 production and IL-2 receptor expression, and regulate cell cycle progression. The final result of CTLA-4 activation is the induction of peripheral tolerance in antigen specific T-cells, by induction of apoptosis or anergy (Figure 1). ${ }^{13-19}$

\section{Preclinical models of checkpoint blockade as tumor immunotherapy}

CTLA-4/B7 interaction blockade with anti-CTLA-4 antibodies was shown to induce rejection of transplanted tumor cell lines in mouse models of colorectal carcinoma, renal carcinoma, lymphoma, prostatic carcinoma, and fibrosarcoma. ${ }^{20-23}$ This antitumor activity appeared to be dependent on the intrinsic immunogenicity of the transplanted tumor cells. The failure of anti-CTLA-4 monotherapy in the less immunogenic tumors (eg, B16 melanoma) led to the exploration of the combination of anti-CTLA-4 with vaccination approaches. ${ }^{1}$ Of particular relevance is the observation that mice carrying a small load of B16 melanoma cells could be successfully treated by combining CTLA-4 blockade with a vaccine consisting of irradiated $\mathrm{B} 16$ cells engineered to produce granulocyte-macrophage colony stimulating factor (GM-CSF). ${ }^{24}$ In the poorly immunogenic B16 murine melanoma model, the combination of CTLA-4 blockade/GM-CSF producing vaccine induced rejection of subcutaneous and metastatic tumors accompanied by autoimmune vitiligo. ${ }^{25}$ 


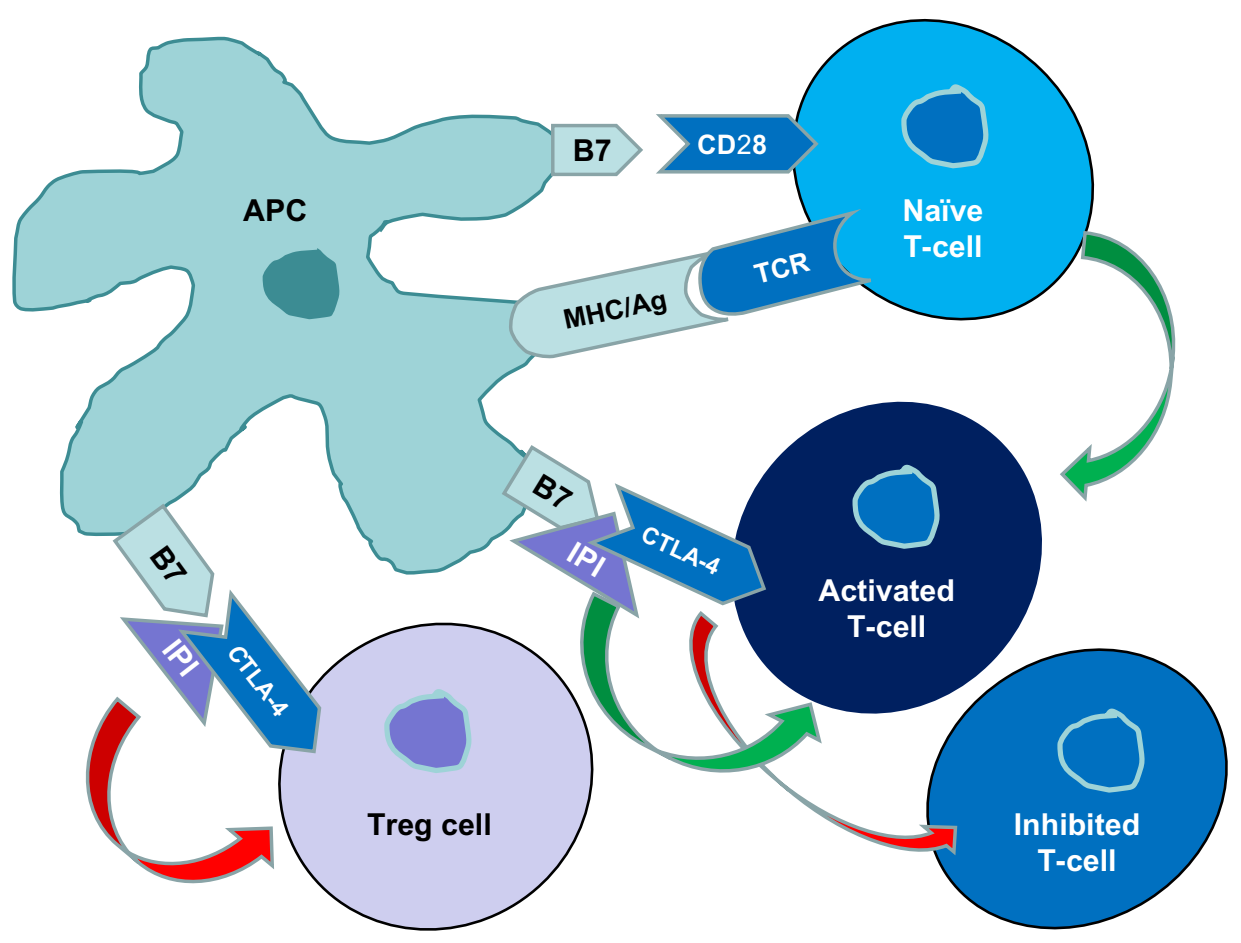

Figure I T-cells are activated by at least two signals between the T-cell and an antigen presenting cell.

Notes: The first signal consists of the presentation of an antigen to the T-cell receptor by the major histocompatibility complex class I or II molecules on an antigen presenting cell. The second costimulatory signal is generated by binding of the CD28 receptor on the T-cell to B7 molecules on the antigen presenting cell. Upregulation of cytotoxic T lymphocyte antigen-4 (CTLA-4), which follows T-cell activation, outcompetes with CD28 for binding to B7 ligands and activates inhibitory signals that turn the activated T-cell into an inhibited T-cell. Ipilimumab, an anti-CTLA-4 antibody, blocks CTLA-4 receptor binding with B7 ligands and allows the T-cell to remain in an activated state, thus enhancing T-cell activity. The second mechanism involved in the CTLA-4 blockade antitumor response is via depletion of tumor induced regulatory T-cells, a suppressive CD4+ T-cell population with an immunosuppressive activity. The green arrow represents stimulation and the red arrow represents inhibition.

Abbreviations: Ag, antigen; APC, antigen presenting cell; CTLA-4, cytotoxic T lymphocyte antigen-4; IPI, ipilimumab; MHC, major histocompatibility complex; TCR, T-cell receptor; Treg, regulatory $\mathrm{T}$-cells.

This depigmentation, similar to that observed in melanoma patients on immunotherapy, suggests that the immune targets for these responses can be represented by normally expressed differentiation antigens. ${ }^{1}$

Hurwitz et al reported that the combination of CTLA-4 blockade and tumor derived GM-CSF resulted in regression of parental mammary carcinoma, despite the ineffectiveness of either treatment alone. ${ }^{26}$ The same group observed that an effective immune response against primary prostate cancer in transgenic mice was elicited using anti-CTLA-4 combined with an irradiated, GM-CSF expressing tumor cell vaccine. ${ }^{27}$ Histopathology revealed that treated mice had a lower tumor grade with accumulation of inflammatory cells in interductal spaces when treated with the combination regimen anti-CTLA-4/vaccine. Vaccination of nontransgenic mice with the same therapeutic strategy resulted in prostatitis, suggesting that the immune response was directed against normal prostate antigens. ${ }^{27} \mathrm{~A}$ similar synergism was observed in preclinical models combining CTLA-4 blockade with xenogeneic DNA, rather than cellular vaccines. ${ }^{28}$
As for the combination of anti-CTLA-4 and peptide vaccines, Davila et al evaluated the role of synthetic oligodeoxynucleotide adjuvants containing unmethylated cytosine-guanine motifs (CpG-ODN) and CTLA-4 blockade in enhancing the antitumor activity of a synthetic peptide. ${ }^{29}$ The combined strategy increased survival of B16 melanoma transplanted mice and was accompanied by the induction of antitumor CD4+ T-cell responses. ${ }^{29}$

\section{Pharmacodynamics and immunity stimulation}

CTLA-4 is inducibly expressed on activated effector CD4+ and CD8+ T-cells and is constitutively expressed on a subset of regulatory T-cells (Tregs). Two main mechanisms are involved in the CTLA-4 blockade induced antitumor response. On one hand, CTLA- 4 acts on activated T-cells to oppose the costimulatory signal provided by the CD28/B7 interaction. This intrinsic model is supported by both in vitro and in vivo observations that $\mathrm{CD} 4+$ and $\mathrm{CD} 8+$ cells that do not express CTLA- 4 have a higher proliferative capacity. ${ }^{30-32}$ The second mechanism is via depletion of tumor induced 
Tregs, a suppressive CD4+ T-cell population that expresses CD25 (the high affinity IL-2 $\alpha$ receptor subunit) and produces immunosuppressive cytokines (eg, IL-10), which inhibit the immune response to tumor associated antigens (Figure 1). ${ }^{1}$

The relative contribution of $\mathrm{CD} 4+$ and $\mathrm{CD} 8+$ effector T-cell versus Treg cell compartments to mediate the effects of CTLA-4 blockade is currently under investigation. In an in vivo model of mouse melanoma expressing human instead of mouse CTLA-4, Peggs et al evaluated the independent contributions of CTLA-4 blockade of each T-cell compartment. ${ }^{33}$ Although blockade of effector T-cells significantly improved tumor protection, blockade of only Treg cells failed to enhance antitumor responses. Concomitant blockade of both effector and Treg cells led to maximal antitumor activity, suggesting that the association of enhancement of effector T-cell activity and concomitant inhibition of Treg function is essential for the therapeutic effects of CTLA-4 blockade. ${ }^{33}$

\section{Clinical data for anti-CTLA-4 antibodies}

CTLA-4 blockade has been shown to be clinically effective in combination with different therapeutic modalities, from chemotherapy to radiotherapy. Different mechanisms may account for the enhancement of the immune response in combination with CTLA-4 blockade, including reduction of tumor burden, increased availability of tumor antigens, and upregulation of costimulatory molecules. When translated to the clinic, treatment with anti-CTLA-4 antibodies resulted in objective tumor responses in a subset of metastatic melanoma and other cancer patients. ${ }^{1}$

Two CTLA-4 antibodies have been tested in clinical trials on patients with metastatic melanoma. Ipilimumab (MDX010) is an IgG1 antibody with a plasma half-life of 12-14 days. Tremelimumab (CP-675,206) is an IgG2 antibody with a plasma half-life of 22 days. ${ }^{34}$

\section{Clinical data for tremelimumab}

In the first Phase I trial with tremelimumab, 39 patients with solid malignancies (melanoma, $\mathrm{N}=34$; renal cell cancer, $\mathrm{N}=4$; colon cancer, $\mathrm{N}=1$ ) received intravenous tremelimumab at seven dose levels. ${ }^{35}$ Dose limiting toxicities and autoimmune phenomena included diarrhea, dermatitis, vitiligo, hypopituitarism, and hyperthyroidism. Two complete responses and two partial responses (maintained $>25$ months) were recorded among 29 patients with measurable disease. Objective tumor responses of metastatic melanoma were noted in a subset of patients starting at the dose of $3 \mathrm{mg} / \mathrm{kg}$ and were more frequent at $15 \mathrm{mg} / \mathrm{kg} .{ }^{35}$
In a Phase I/II trial, 28 patients with metastatic melanoma received monthly infusion of tremelimumab at 3,6 , or $10 \mathrm{mg} / \mathrm{kg}$ for up to 12 months. ${ }^{36}$ During Phase II, 89 patients received tremelimumab $10 \mathrm{mg} / \mathrm{kg}$ every month or $15 \mathrm{mg} / \mathrm{kg}$ every 3 months. No dose limiting toxicity was observed in Phase I. In Phase II, 8 (10\%) of 84 patients experienced objective antitumor responses: the best overall objective response was one complete response and three partial responses in each dosing regimen and most responses were durable for a duration of up to $>30$ months. The results of this study suggested that the $15 \mathrm{mg} / \mathrm{kg}$ every 3 month schedule was preferred based on lower toxicity while maintaining tumor response. ${ }^{36}$

A large randomized controlled Phase III clinical trial on 665 metastatic melanoma patients comparing tremelimumab (15 mg/kg every 3 months) with dacarbazine or temozolomide recorded a median overall survival of 11.7 months with tremelimumab versus 10.7 months for conventional chemotherapy, without a statistically significant difference. ${ }^{35}$ The proportion of partial responses was similar in the two groups $(1.5 \%$ versus $1.8 \%)$.

\section{Clinical data for ipilimumab Phase I/II studies}

Ipilimumab was used in several Phase I clinical trials in different tumor types, including prostate cancer, ovarian carcinoma, renal carcinoma, and melanoma. Based on preclinical data the initial dose of $3 \mathrm{mg} / \mathrm{kg}$ was chosen. Dose limiting toxicities were observed in dose escalation trials but a maximum tolerated dose was not established. ${ }^{37-39}$

Forty-six pretreated, HLA-A0201 negative metastatic melanoma patients received ipilimumab at the dose of $3 \mathrm{mg} / \mathrm{kg}(\mathrm{N}=23)$ or $5 \mathrm{mg} / \mathrm{kg}(\mathrm{N}=23)$, then the dose was escalated to a maximum of $9 \mathrm{mg} / \mathrm{kg}$. Sixteen patients $(35 \%)$ experienced grade $3 / 4$ autoimmune toxicity and five patients (11\%) had a partial response according to Response Evaluation Criteria in Solid Tumors (RECIST) criteria after receiving the drug at $9 \mathrm{mg} / \mathrm{kg}^{39}$

In a Phase I/II study on 88 advanced melanoma patients, single doses of ipilimumab up to $20 \mathrm{mg} / \mathrm{kg}$, or multiple doses up to $5 \mathrm{mg} / \mathrm{kg}$, or multiple doses up to $10 \mathrm{mg} / \mathrm{kg}$ were administered. ${ }^{40}$ Although a maximum tolerated single dose was not established, multiple dosing at $10 \mathrm{mg} / \mathrm{kg}$ resulted in better objective responses and durable stable diseases than was observed in the single high dose group. ${ }^{40}$

In 2008, Hodi et al reported the data on clinical and immunologic effects of anti-CTLA-4 antibodies after vaccination with irradiated, autologous tumor cells engineered to secrete GM-CSF (GVAX) in advanced melanoma and ovarian 
cancer. ${ }^{41}$ In contrast to other more intensive regimens, no grade $3 / 4$ toxicities were observed in the melanoma cohort. By standard RECIST, three patients had a partial response. Biopsies of metastatic lesion were performed in six melanoma patients and revealed minimal to extensive tumor necrosis. All samples with extensive tumor necrosis contained a dense CD8+ cell infiltrate. Tumor necrosis was inversely related to the number of intratumoral FoxP3+ Treg cells $(P<0.0001)$ and linearly related to the natural logarithm of the ratio of infiltrating CD8+/FoxP3 Treg cells $(P<0.0001)$, corroborating other observations that the combination treatment of GVAX and anti-CTLA-4 in B16 murine melanoma modulated the balance of antitumor CD8+ and Treg cells. ${ }^{27}$ Two of nine ovarian cancer patients developed a grade 3 gastrointestinal toxicity and an objective response was observed in four patients (one partial response and three stable disease). Although only a small number of patients was recruited, the results of this study suggested that the combined treatment with immune stimulating agents may enhance the antitumor activity exerted by anti-CTLA- 4 antibodies. ${ }^{41}$

Recently, data regarding the combination of ipilimumab with nivolumab, an antibody against the programmed death 1 receptor, were published by Wolchok et al. ${ }^{42}$ Nivolumab had already shown durable tumor regression in a Phase I trial. ${ }^{43}$ In the Phase I trial by Wolchok et al, ${ }^{42}$ successive cohorts of advanced melanoma patients were treated with escalating doses of intravenous nivolumab and ipilimumab administered concurrently every 3 weeks for four doses, followed by nivolumab alone every 3 weeks for four doses (concurrent regimen group). The combined treatment was subsequently administered every 12 weeks for up to eight doses. In a sequenced regimen, patients previously treated with ipilimumab received nivolumab every 2 weeks for up to 48 doses. A total of 53 patients received concurrent therapy with nivolumab and ipilimumab, and 33 received sequenced treatment. The objective response rate for all patients in the concurrent regimen group was $40 \%$. Evidence of clinical activity was observed in $65 \%$ of patients. Grade 3 or 4 adverse events related to therapy were recorded in $53 \%$ of patients in the concurrent regimen group. Among patients in the sequenced regimen group, $18 \%$ experienced grade 3 or 4 adverse events related to therapy and the objective response rate was $20 \%$ (Table 1). ${ }^{42}$

\section{Phase II studies}

In a Phase II clinical trial, 217 pretreated, unresectable stage III/IV melanoma patients were randomly assigned to receive $0.3(\mathrm{~N}=73), 3(\mathrm{~N}=72)$, or $10(\mathrm{~N}=72) \mathrm{mg} / \mathrm{kg}$ ipilimumab every 3 weeks four times, then every 3 months until progression. ${ }^{44}$ A dose dependent effectiveness in the clinical activity was recorded with the dose of $10 \mathrm{mg} / \mathrm{kg}$ showing a best overall response rate (complete and partial responses) of $11.1 \%$.

Table I Selected Phase I and II clinical studies with ipilimumab in melanoma patients

\begin{tabular}{|c|c|c|c|c|c|c|}
\hline Phase & $\begin{array}{l}\text { Number of } \\
\text { patients }\end{array}$ & Regimen and arms & Response & Criteria & Author & Reference \\
\hline 1 & 46 & $\begin{array}{l}\text { Ipilimumab 3-9 } \mathrm{mg} / \mathrm{kg} \text {, with } \\
\text { intrapatient dose escalation }\end{array}$ & 5 PR (II\%) & RECIST & Maker et al & 39 \\
\hline \multirow[t]{3}{*}{ I/II } & 88 & $\begin{array}{l}\text { Group A, ipilimumab single } \\
\text { dose: up to } 20 \mathrm{mg} / \mathrm{kg}\end{array}$ & I PR, 3 SD & RECIST & Weber et al & 40 \\
\hline & & $\begin{array}{l}\text { Group A, ipilimumab multiple } \\
\text { doses: up to } 5 \mathrm{mg} / \mathrm{kg}\end{array}$ & I PR, 4 SD & & & \\
\hline & & $\begin{array}{l}\text { Group B, ipilimumab multiple } \\
\text { doses up to } 10 \mathrm{mg} / \mathrm{kg}\end{array}$ & I PR, I CR, 7 SD & & & \\
\hline 1 & 11 & $3 \mathrm{mg} / \mathrm{kg}+\mathrm{GVAX}$ & 3 PR, 5 SD & RECIST & Hodi et al & $4 I$ \\
\hline \multirow[t]{3}{*}{ II } & 217 & Ipilimumab $0.3 \mathrm{mg} / \mathrm{kg}$ & I0 SD (BORR 0\%) & RECIST & Wolchok et al & 44 \\
\hline & & Ipilimumab 3 mg/kg & 3 PR, I6 SD (BORR 4.2\%) & & & \\
\hline & & Ipilimumab 10 mg/kg & $2 \mathrm{CR}, 6 \mathrm{SD}, \mathrm{I} 3 \mathrm{SD}$ (BORR II.I\%) & & & \\
\hline II & 155 & Ipilimumab 10 mg/kg & 5 PR, 33 SD (BORR 5.8\%) & RECIST & O’Day et al & 45 \\
\hline \multirow[t]{2}{*}{ II } & 115 & Ipilimumab 10 mg/kg + budesonide & I CR, 6 PR (BORR I2\%) & RECIST & Weber et al & 46 \\
\hline & & Ipilimumab 10 mg/kg + placebo & 0 CR, 9 PR (BORR I6\%) & & & \\
\hline II & 72 & Ipilimumab 10 mg/kg & $\begin{array}{l}0 \mathrm{CR}, 6 \mathrm{PR}, 4 \mathrm{SD} \text { (mWHO), } \\
9 \mathrm{SD} \text { (irRC) }\end{array}$ & $\begin{array}{l}\mathrm{mWHO} \\
\operatorname{irRC}\end{array}$ & Margolin et al & 48 \\
\hline II & 86 & $\begin{array}{l}\text { Ipilimumab } 10 \mathrm{mg} / \mathrm{kg}+ \\
\text { fotemustine } 100 \mathrm{mg} / \mathrm{m}^{2}\end{array}$ & $\begin{array}{l}6 \mathrm{irCR}, 19 \mathrm{irPR}, 15 \mathrm{irSD}, \\
40 \mathrm{irDC}(46.5 \%)\end{array}$ & irRC & $\begin{array}{l}\text { Di Giacomo } \\
\text { et al }\end{array}$ & 49 \\
\hline
\end{tabular}

Abbreviations: BORR, best overall response rate; CR, complete response; GVAX, autologous tumor cells engineered to secrete granulocyte-macrophage colony stimulating factor; irCR, immune related complete response; irDC, immune related disease control; irPR, immune related partial response; irRC, immune related response criteria; irSD, immune related stable disease; $\mathrm{mWHO}$, modified World Health Organization criteria; PR, partial response; RECIST, Response Evaluation Criteria in Solid Tumors; SD, stable disease. 
Although the study design had no power to detect differences in overall survival, a positive trend for both overall survival and progression free interval was noted for ipilimumab at the highest dose. ${ }^{44}$ This was the first randomized clinical trial to evaluate the dose responsiveness of ipilimumab. The most common grade 3-4 adverse events were gastrointestinal immune related adverse events (irAEs) (eleven in the $10 \mathrm{mg}$ / $\mathrm{kg}$ group and two in the $3 \mathrm{mg} / \mathrm{kg}$ group) and diarrhea.

Another Phase II study evaluated the safety and activity of ipilimumab in previously treated patients with stage III (unresectable)/stage IV melanoma. Patients $(\mathrm{N}=155)$ received ipilimumab at the dose of $10 \mathrm{mg} / \mathrm{kg}$ every 3 weeks for four cycles followed by maintenance therapy every 3 months. ${ }^{45}$ The best overall response rate was 5.8\% (five partial responses, no complete response) with 1- and 2-year survival rates of $47.2 \%$ and $32.8 \%$. Even if this study was not designed to show an improvement in overall survival, encouraging survival outcomes were observed. Adverse events were mainly irAEs involving the skin and gastrointestinal tract. ${ }^{45}$

A third Phase II study was a randomized study in which 115 untreated and previously treated patients received ipilimumab at the dose of $10 \mathrm{mg} / \mathrm{kg}$, every 3 weeks for four doses, with and without prophylactic budesonide. ${ }^{46}$ The best overall response rate ranged from $12.7 \%$ (ipilimumab plus budesonide group) to $15.8 \%$ (ipilimumab plus placebo group), and the median overall survival was 17.7 and 19.3 months, respectively. Budesonide did not significantly affect the incidence of grade $\geq 2$ diarrhea. ${ }^{46}$ Follow-up analyses of Phase II trials in patients with advanced melanoma showed a 1-year survival rate of around $25.5 \% .{ }^{47}$

An open-label Phase II trial prospectively evaluated ipilimumab activity in patients with active melanoma brain metastases. ${ }^{48}$ Patients received four doses of $10 \mathrm{mg} / \mathrm{kg}$ of ipilimumab every 3 weeks. Seventy-two patients were stratified into two groups: those in arm $\mathrm{A}(\mathrm{N}=51)$ were neurologically asymptomatic and were not receiving corticosteroids at the beginning of the study and patients in $\operatorname{arm} \mathrm{B}(\mathrm{N}=21)$ were symptomatic and on a stable dose of steroids. Results suggested that ipilimumab had a similar level of activity both in brain and in noncentral nervous system metastases with a disease control rate of $24 \%$ and $27 \%$, respectively, for the patients in arm A. ${ }^{48}$ Responses in the brain were durable, with a median duration $>15$ months. ${ }^{17}$

In the NIBIT-M1 Phase II trial, 86 patients with unresectable stage III/IV melanoma received ipilimumab $10 \mathrm{mg} / \mathrm{kg}$ every 3 weeks for four doses and fotemustine $100 \mathrm{mg} / \mathrm{m}^{2}$ weekly for 3 weeks and, and subsequently every 3 weeks. ${ }^{49}$ Patients with a confirmed clinical response were eligible for maintenance treatment from week 24 , with ipilimumab every 12 weeks and fotemustine every 3 weeks. To note, 20 patients had asymptomatic brain metastases at baseline. Forty patients achieved disease control $(46.5 \%, 95 \%$ confidence interval $[\mathrm{CI}]: 35.7-57.6)$, as did ten patients with brain metastases (50\%). After a median follow-up of 8.3 months, median progression free survival was 5.3 months (95\% CI: 3.5-7.1) and the 1-year overall survival was $51.8 \%$ (95\% CI: $37.5-66.1)$. Forty-seven patients ( $55 \%$ ) had grade 3 or 4 treatment related adverse events, of which the most common was myelotoxicity (Table 1$).{ }^{49}$

\section{Phase III studies}

The most promising results for ipilimumab were published by Hodi et al in $2010 .{ }^{50}$ In this Phase III study, ipilimumab administered with or without a glycoprotein 100 (gp100) peptide vaccine was compared with gp100 alone in previously treated advanced melanoma patients. A total of 676 HLA-A0201 positive patients with unresectable stage III/IV melanoma were randomly assigned to receive ipilimumab plus gp100 ( $\mathrm{N}=403)$, ipilimumab alone $(\mathrm{N}=137)$, or gp100 alone $(\mathrm{N}=136)$. In the induction phase, ipilimumab at $3 \mathrm{mg} / \mathrm{kg}$ was administered with or without gp100 every 3 weeks for up to four treatments. Eligible patients could receive reinduction therapy. The primary endpoint was overall survival. The median overall survival was 10.0 months for patients receiving ipilimumab plus gp100 and 6.4 months for patients receiving gp100 alone (hazard ratio for death $0.68 ; P<0.001$ ). The median overall survival with ipilimumab alone was 10.1 months (hazard ratio for death in comparison with gp 100 alone $0.66, P=0.003$ ). No difference in overall survival was detected between the ipilimumab groups. Grade 3 or 4 irAEs occurred in $10 \%-15 \%$ of patients treated with ipilimumab and in $3 \%$ of those treated with gp 100 alone. Fourteen deaths related to the study drugs $(2.1 \%)$ were recorded, and seven were associated with irAEs. The authors concluded that ipilimumab, with or without gp100, when compared with gp100 alone, improved overall survival in patients with previously treated metastatic melanoma. ${ }^{50}$

Remarkably, about $70 \%$ of patients enrolled in this trial had M1c stage (with pretreated central nervous system metastases not being an exclusion criteria), and 37\% had elevated serum lactic dehydrogenase (LDH) levels. ${ }^{50}$ All these factors, considered predictors of poor survival, did not seem to affect patients' response rates. ${ }^{17,34}$ The survival kinetics showed a delayed separation for overall survival curves, which was consistent with a building cellular immune response followed by changes in tumor burden and overall 
survival. ${ }^{51}$ Even though the study did not include a maintenance dosing phase, ipilimumab reinduction was evaluated. Patients ( $\mathrm{N}=40)$ showing a confirmed objective response or stable disease $\geq 3$ months were reinduced with additional courses of anti-CTLA-4 following disease progression, with a disease control rate of up to $67 \% .^{52}$

In the study by Hodi et al, patients were selected for HLA-A0201 positive status based on the mechanism of action of gp $100 .^{50}$ However, the activity of anti-CTLA-4 antibody may be independent of HLA-A0201status, given that anti-CTLA-4 targets the TCR-major histocompatibility complex I/II checkpoint. ${ }^{17}$ An unconventional aspect of the study by Hodi et al is that the control arm did not consist of dacarbazine treated patients, as was the case in previous clinical Phase III trials, but instead was a vaccine treated group. Indeed, the best overall response rate was higher in the antiCTLA-4 group than in the combination group (anti-CTLA-4 plus gp 100). However, the combination group did perform better than the group receiving the vaccine alone, suggesting that it is unlikely that the vaccine could have been harmful in the study by Hodi et al. ${ }^{50}$ This observation was further corroborated by recent results from another Phase III study on 185 HLA-A0201stage IV or advanced stage III melanoma patients who were randomly assigned to receive IL-2 alone or gp 100:209-217(210M) plus incomplete Freund' adjuvant (Montanide ISA-51) plus IL-2..$^{53}$ The vaccine/IL-2 group had a significant improvement in overall clinical response ( $16 \%$ versus $6 \%, P=0.03$ ) and a longer progression free and median overall survival, when compared with the IL-2 group. ${ }^{53}$ Although, in the study by Hodi et al, ${ }^{50}$ the difference in the median overall survival in the two groups receiving antiCTLA- 4 versus the gp 100 alone group was only 4 months, the true impact of the anti-CTLA treatment was in the long-term benefit which was observed in a subset of patients. ${ }^{2}$

Long-term follow-up data of responder patients were recently reviewed. Prieto et al evaluated 177 melanoma patients treated with ipilimumab in three clinical trials from 2002 to $2005 .{ }^{51}$ In protocol 1, 56 patients received ipilimumab with gp100 peptides. In protocol 2, 36 patients received ipilimumab with IL-2. In protocol 3, 85 patients received ipilimumab with intrapatient dose escalation and were randomized to receive gp 100 peptide. Long-term follow-up showed that the median survival was 14 months for protocol 1 (median follow-up 92 months; 5-year survival rate 13\%), 16 months for protocol 2 (median follow-up 84 months; 5 -year survival rate $25 \%$ ), and 13 months for protocol 3 (median follow-up 71 months; 5-year survival rate 23\%). These results were consistent with durable tumor regressions (past 6 years) induced by CTLA-4 blockade in subsets of metastatic melanoma patients and highlighted the importance of identifying valuable markers to predict this outcome. ${ }^{54}$

In 2011 the results of a second Phase III trial on 502 patients with previously untreated metastatic melanoma were published by Robert et al. ${ }^{55}$ Patients were randomly assigned to ipilimumab $(10 \mathrm{mg} / \mathrm{kg})$ plus dacarbazine $\left(850 \mathrm{mg} / \mathrm{m}^{2}\right)$ or dacarbazine $\left(850 \mathrm{mg} / \mathrm{m}^{2}\right)$ plus placebo. Treatment was given every 3 weeks for four cycles and followed by dacarbazine alone through week 22. Responding patients (stable disease or objective response) could receive ipilimumab or placebo every 3 months as maintenance therapy. Overall survival was significantly longer in the ipilimumab plus dacarbazine group than in the dacarbazine plus placebo group (11.2 months versus 9.1 months, respectively), with higher survival rates in the ipilimumab plus dacarbazine group at 1,2 , and 3 years (hazard ratio for death, $0.72 ; P<0.001$ ). Grade 3 and 4 adverse events occurred in $56.3 \%$ of patients in the ipilimumab plus dacarbazine group compared with $27.5 \%$ treated with dacarbazine and placebo $(P<0.001)$. The most common study drug adverse events were those classified as irAEs, and were observed in $77.7 \%$ of patients in the ipilimumab plus dacarbazine arm. The most common grade 3 or 4 irAEs were immune mediated hepatitis, and were observed in 78 patients in the ipilimumab plus dacarbazine group (31.6\%). Grade 3 or 4 immune mediated colitis was observed in 12 patients in the ipilimumab plus dacarbazine arm (4.9\%). No gastrointestinal perforations were reported. No drug related deaths were reported in the ipilimumab plus dacarbazine group. To note, more than $50 \%$ of patients had visceral metastases, elevated LDH levels, or both. Durable objective responses were recorded with a median duration of best overall response of 19.3 months in the ipilimumab plus dacarbazine group compared to 8.1 months in the dacarbazine group. In the ipilimumab plus dacarbazine group, an estimated $28.5 \%$ of patients were alive at 2 years and $20.8 \%$ at 3 years (versus $17.9 \%$ and $12.2 \%$, respectively, in the dacarbazine group)..$^{55}$

In March 2011, ipilimumab became the first drug to receive Food and Drug Administration approval to treat patients with late stage melanoma that has spread or that cannot be removed by surgery. ${ }^{56}$

\section{Current trials with ipilimumab}

Combinations of ipilimumab with other therapeutic approaches are currently under investigation in several clinical trials. Phase II studies evaluating toxicity and efficacy of ipilimumab combined with chemotherapy (ie, dacarbazine, 
carboplatin plus paclitaxel), or immunotherapy (ie, BCG [Bacillus of Calmette-Guèrin], dendritic cell vaccine, interferon $\alpha-2 b$ ), or radiotherapy are currently recruiting patients. ${ }^{57}$ New target therapies, such as vemurafenib, a BRAF inhibitor, were tested in combination with ipilimumab, either as combined or sequential treatments, but no published data are available as yet on toxicity and outcome of such a combination. An ongoing study aims at the evaluation of ipilimumab as monotherapy in the adjuvant setting for resected, high risk stage III melanoma patients (CA184-029). ${ }^{17}$

\section{Immune related response criteria}

In Phase II studies aimed at evaluating the effect of a drug without a concurrent control group, the objective response was the most frequently used primary endpoint. ${ }^{58}$ Nevertheless, objective response criteria according to World Health Organization (WHO) or RECIST were developed to define the effects of chemotherapy; therefore, they may not be adequate for agents with other mechanisms of action, ie, antiangiogenic drugs, cytokines, or immunomodulating agents. Besides the conventional patterns of partial/complete response and stable disease (decrease or stability in target lesion dimensions, without appearance of new lesions), ipilimumab has also shown activity after an increase in tumor burden, as demonstrated in specimens of regressing tumors during therapy with anti-CTLA-4. ${ }^{51}$ These tumors became infiltrated with CD8+ lymphocytes, leading to tumor cell killing and eventual regression of metastases, detectable by objective response criteria. ${ }^{41,59}$ This delayed, radiologically assessed, clinical activity may reflect the dynamics of the immune system. ${ }^{51}$

In 2009, a new set of response criteria for tumor immunotherapy was proposed, which was termed immune related response criteria (irRC) ${ }^{60}$ The irRC were derived from the WHO and RECIST criteria and were defined on the basis of the results from Phase II studies with ipilimumab. ${ }^{17}$ Four distinct response patterns were described: immediate response, durable stable disease, response after tumor burden increase, and response in the presence of new lesions. All these patterns were associated with an improved overall survival compared to patients with progressive disease as assessed by standard criteria. While the first two patterns are conventional, the latter two are specifically recognized with immunotherapeutic agents and may identify potential subsets of responding patients who otherwise would be considered progressive disease according to standard criteria. In particular, the use of irRC may avoid early discontinuation of immunotherapeutic regimens in patients who may eventually benefit from treat- ment, ie, those patients where tumor infiltration may mimic a progressive disease. ${ }^{51,60}$

\section{irAEs}

Vitiligo in mice treated with anti-CTLA-4 antibodies in combination with a GM-CSF expressing tumor cell vaccine evidenced the ability of CTLA-4 blockade to break peripheral immune tolerance. ${ }^{27}$ Histological evaluation of depigmented lesions revealed infiltration of polymorphonuclear cells and deposition of antibody. ${ }^{61}$ Prostatitis in a prostate cancer model was also observed. ${ }^{26}$ In early clinical studies "autoimmunity" adverse events (ie, dermatitis, enterocolitis, hepatitis, uveitis, hypophysitis, thyroiditis) consistent with anti-CTLA-4 dependent breaking tolerance to self antigens were reported. ${ }^{38,62}$ These unique side effects of CTLA-4 blockade were associated with prolonged survival in patients with metastatic melanoma and were later termed irAEs. ${ }^{37}$ Even if in most cases irAEs were reversible in clinical trials with ipilimumab, irAEs may be severe and life threatening. Grade 3/4 diarrhea/colitis was the most frequently reported adverse event in clinical trials and in a few cases resulted in death due to bowel perforation. ${ }^{17,34}$ In one Phase III clinical trial, ${ }^{17}$ grade $3 / 4$ irAEs occurred in $10 \%-15 \%$ of patients treated with anti-CTLA-4 antibodies and 14 deaths related to the study drugs were reported. Of these, seven were associated with irAEs. Four of these seven patients died of bowel perforation. ${ }^{17,50}$

Grade 3/4 irAEs must be treated with high dose corticosteroids and other immunosuppressive therapies in the most severe cases. Corticosteroids used to treat irAEs do not appear to affect the efficacy of anti-CTLA-4 therapy. ${ }^{63}$

The relationship between irAEs and antitumor activity associated with anti-CTLA-4 therapy was explored in clinical studies. A higher frequency of these toxicities, and especially grade 3/4 irAEs, was observed in responding patients. ${ }^{38,63}$ In the double blind, placebo controlled, Phase II study by Weber et al, the disease control rate was higher in patients with grade $3 / 4$ irAEs than in patients with grade $0 / 2$ irAEs, although many patients with grade $1 / 2$ irAEs also experienced a clinical benefit. ${ }^{46}$ Nonetheless, data from other trials or pooled analyses were not conclusive and so far, the relationship between severity of irAEs and clinical response to anti-CTLA-4 therapy is not definitive.

\section{Potential biomarkers for clinical response and survival}

In a single institution experience, 53 advanced melanoma patients received ipilimumab $10 \mathrm{mg} / \mathrm{kg}$ every 3 weeks 
for four doses. ${ }^{64}$ Patients with an absolute lymphocyte count $($ ALC) $\geq 1,000$ cells $/ \mu \mathrm{L}$ after two anti-CTLA-4 doses had a significantly improved clinical benefit rate (51\% versus $0 \% ; P=0.01$ ) and median overall survival (11.9 versus 1.4 months; $P<0.001$ ) when compared with those with an ALC $<1,000$ cells $/ \mu L{ }^{64}$ The rationale for the correlation between ALC and antitumor effect may reflect the immunologic activity of ipilimumab. Presumably, an ALC value of 1,000 cells/ $\mu \mathrm{L}$ represents a threshold for the immune system to be adequately stimulated by ipilimumab to mediate objective responses. Further investigations are needed to confirm if ALC represents a biomarker for tumor response to help identify patients who may not benefit from anti-CTLA-4 therapy. ${ }^{64}$

Interestingly, other well known prognostic factors in advanced melanoma, such as serum LDH level, do not seem to impact on clinical benefit in patients under anti-CTLA-4 therapy. ${ }^{50}$

In a pooled analysis of four Phase II trials, peripheral blood lymphocyte subsets were analyzed from 35 advanced melanoma patients receiving ipilimumab $10 \mathrm{mg} / \mathrm{kg}$ every 3 weeks for four doses. ${ }^{65}$ Patients could receive a maintenance treatment in case of disease control. Interestingly, increased CD8+ T-cells were associated with improved clinical outcome, while absolute change in CD4+ CD25+T-cells did not seem to differ among the clinical outcome groups. ${ }^{65}$

In a recent report of a Phase II trial, 75 stage IIIC/IV, surgically rendered free of disease, melanoma patients received ipilimumab either at $3 \mathrm{mg} / \mathrm{kg}(\mathrm{N}=25)$ or $10 \mathrm{mg} / \mathrm{kg}$ $(\mathrm{N}=50)$ every 6-8 weeks for 12 months. ${ }^{66}$ Eligible patients received further maintenance treatments. In HLA-A0201 positive patients, anti-CTLA-4 infusions were combined with subcutaneous injections of a multipeptide vaccine containing tyrosinase, gp100, and Mart-1. Interestingly, patients whose baseline C-reactive protein (CRP) level was $2 \mathrm{mg} / \mathrm{L}$ or greater had a significantly improved freedom from relapse. These results were in contrast with a previous study by Marshall et al who performed blood analyses from 525 patients in a randomized Phase III trial comparing a different CTLA-4 blocking antibody, tremelimumab, versus dacarbazine or temozolomide in patients with advanced melanoma. In this study, patients with a low baseline CRP ( $\leq 1.5$ times the upper normal limit) experienced an improved overall survival, while patients with higher baseline level of CRP apparently did not benefit from tremelimumab when compared to chemotherapy. ${ }^{66}$

In a randomized, double blind, Phase II study aimed at identifying biomarkers of response and toxicity in advanced melanoma patients, 82 patients were induced with ipilimumab either at 3 or $10 \mathrm{mg} / \mathrm{kg}$, every 3 weeks, for four doses and could receive maintenance therapy from week $24 .{ }^{67}$ Candidate biomarkers were evaluated in tumor biopsies collected before treatment started and up to 72 hours after the second dose of ipilimumab. Immunohistochemistry and histology on tumor biopsies revealed significant associations between clinical outcome and high baseline levels of FoxP3 $(P=0.0014)$ and indoleamine 2,3-dioxygenase $(P=0.0012)$, and between clinical activity and increase in tumor infiltrating lymphocytes within 3 weeks after the first administration $(P=0.005)$. Microarray analysis of mRNA from tumor samples obtained pre- and post-treatment showed significantly increased expression of several immune related genes (immunoglobulins, granzyme B, perforin-1, granulysin, CD8 beta-subunit, and TCR- $\alpha$ and $-\beta$ subunits) and decreases in expression of genes implicated in tumor progression (tyrosinase-related protein-2). ${ }^{67}$ Interestingly, FoxP3 is expressed by immunosuppressive Tregs, which play a major role in suppression of the immune response in the tumor microenvironment. Indoleamine 2,3-dioxygenase is an immune regulatory enzyme exerting a pro-apoptotic activity on T-cells as well as melanoma cells. ${ }^{17,68}$ The prognostic significance of tumor infiltrating lymphocytes has been associated with a favorable prognosis both in melanoma and other cancer types. ${ }^{69}$

Another important biomarker may be the expression on the inducible costimulator (ICOS) molecule, a member of the immunoglobulin gene family, on T-cells. ${ }^{70}$ ICOS was associated with clinical outcome in a small cohort of patients with localized urothelial carcinoma treated with ipilimumab in a preoperative setting and the ICOS (inducible costimulator)/ ICOSL pathway was found to be necessary for the optimal therapeutic effect of anti-CTLA-4. ${ }^{71,72}$

\section{Conclusion}

Ipilimumab is a new generation immunotherapeutic agent that has shown activity in terms of disease free and overall survival in metastatic melanoma patients. Ipilimumab was approved by the Food and Drug Administration in March 2011 to treat patients with late stage melanoma that has spread or that cannot be removed by surgery. In July 2011, the European Commission approved ipilimumab for the treatment of adult patients with advanced melanoma as second line treatment at the dose of $3 \mathrm{mg} / \mathrm{kg}$. Ipilimumab has not yet been approved as maintenance therapy, but patients who have progressed after an initial benefit may be retreated if clinically indicated. 
Ipilimumab treatment has been associated with severe irAEs due to T-cell activation and proliferation. Most of these serious adverse effects are associated with the gastrointestinal tract and include severe diarrhea and colitis. Some cases of deaths have been reported, most frequently due to bowel perforation. These unique side effects of CTLA-4 blockade were sometimes associated with prolonged survival.

Because patients on anti-CTLA-4 therapy may experience delayed responses or durable stable disease after an initial apparent disease progression (due to tumor T-cell infiltration and inflammation), irRC is now used to better analyze additional responses that would otherwise be classified as progressive disease according to RECIST or WHO criteria. The introduction of irRC may avoid early discontinuation of immunotherapeutic regimens in patients who may eventually benefit from treatment.

An open issue concerns the optimal dose of ipilimumab, with the dose of $10 \mathrm{mg} / \mathrm{kg}$ being presently underway in clinical trials. Data on markers predictive for benefit from treatment with ipilimumab are still not known. An increase in ALC may be a marker of immune stimulation and occurrence of immune related reactions may accompany clinical effectiveness. Ipilimumab is currently under investigation in combination with other treatments, such as chemotherapy, target agents, radiotherapy, and other immunotherapeutic regimens. ${ }^{73}$

\section{Disclosure}

The authors report no conflict of interest in this work.

\section{References}

1. Korman AJ, Peggs KS, Allison JP. Checkpoint blockade in cancer immunotherapy. Adv Immunol. 2006;90:297-339.

2. Hwu P. Treating cancer by targeting the immune system. $N$ Engl J Med. 2010 19;363(8):779-781.

3. Chambers CA, Kuhns MS, Egen JG, Allison JP. CTLA-4-mediated inhibition in regulation of $\mathrm{T}$ cell responses: mechanisms and manipulation in tumor immunotherapy. Annu Rev Immunol 2001;19:565-594.

4. Linsley PS, Greene JL, Tan P, et al. Coexpression and functional cooperation of CTLA-4 and CD28 on activated T lymphocytes. J Exp Med. 1992;176(6):1595-1604.

5. Krummel MF, Allison JP. CD28 and CTLA-4 have opposing effects on the response of T cells to stimulation. J Exp Med. 1995;182(2):459-465.

6. Kearney ER, Walunas TL, Karr RW, et al. Antigen-dependent clonal expansion of a trace population of antigen-specific CD4+ T cells in vivo is dependent on CD28 costimulation and inhibited by CTLA-4. J Immunol. 1995;155(3):1032-1036.

7. Walunas TL, Lenschow DJ, Bakker CY, et al. CTLA-4 can function as a negative regulator of T cell activation. Immunity. 1994;1(5):405-413.

8. Krummel MF, Sullivan TJ, Allison JP. Superantigen responses and costimulation: CD28 and CTLA-4 have opposing effects on T cell expansion in vitro and in vivo. Int Immunol. 1996;8(4):519-523.

9. Tivol EA, Borriello F, Schweitzer AN, Lynch WP, Bluestone JA, Sharpe AH. Loss of CTLA-4 leads to massive lymphoproliferation and fatal multiorgan tissue destruction, revealing a critical negative regulatory role of CTLA-4. Immunity. 1995;3:541-547.
10. Waterhouse P, Penninger JM, Timms E, et al. Lymphoproliferative disorders with early lethality in mice deficient in CTLA-4. Science. 1995;270:985-988.

11. Chambers CA, Cado D, Truong T, Allison JP. Thymocyte development is normal in CTLA-4-deficient mice. Proc Natl Acad Sci USA. 1997;94:9296-9301.

12. Waterhouse P, Bachmann MF, Penninger JM, Ohashi PS, Mak TW. Normal thymic selection, normal viability and decreased lymphoproliferation in T cell receptor-transgenic CTLA-4-deficient mice. Eur J Immunol. 1997;27(8):1887-1892.

13. Melero I, Hervas-Stubbs S, Glennie M, Pardoll DM, Chen L. Immunostimulatory monoclonal antibodies for cancer therapy. Nat Rev Cancer. 2007;7(2):95-106.

14. Thompson CB, Allison JP. The emerging role of CTLA-4 as an immune attenuator. Immunity. 1997;7(4):445-450.

15. de la Cruz-Merino L, Grande-Pulido E, Albero-Tamarit A, CodesManuel de Villena ME. Cancer and immune response: old and new evidence for future challenges. Oncologist. 2008;13(12):1246-1254.

16. Alegre ML, Fallarino F. Mechanisms of CTLA-4-Ig in tolerance induction. Curr Pharm Des. 2006;12(2):149-160.

17. Hoos A, Ibrahim R, Korman A, et al. Development of ipilimumab: contribution to a new paradigm for cancer immunotherapy. Semin Oncol. 2010;37(5):533-546.

18. Krummel MF, Allison JP. CTLA-4 engagement inhibits IL-2 accumulation and cell cycle progression upon activation of resting T cells. $J$ Exp Med. 1996;183(6):2533-2540.

19. Brunner MC, Chambers CA, Chan FK, Hanke J, Winoto A, Allison JP. CTLA-4-Mediated inhibition of early events of $\mathrm{T}$ cell proliferation. J Immunol. 1999;162(10):5813-5820.

20. Yang Y, Zou J, Mu J, et al. Enhanced induction of antitumor T-cell responses by cytotoxic $\mathrm{T}$ lymphocyte associated molecule-4 blockade: the effect is manifested only at the restricted tumor-bearing stages. Cancer Res. 1997;57:4036-4041.

21. Shrikant P, Khoruts A, Mescher MF. CTLA-4 blockade reverses CD8C T cell tolerance to tumor by a CD4CT cell and IL-2 dependent mechanism. Immunity. 1999;11:483-493.

22. Sotomayor EM, Borrello IM, Tubb E, Allison JP, Levitsky HI. In vivo blockade of CTLA-4 enhances the priming of responsive T-cells but fails to prevent the induction of tumor antigen-specific tolerance. Proc Natl Acad Sci US A. 1999;96(20):11476-11481.

23. Leach DR, Krummel MF, Allison JP. Enhancement of antitumor immunity by CTLA-4 blockade. Science. 1996;271(5256):1734-1736.

24. Dranoff G, Jaffee E., Lazenby A, et al. Vaccination with irradiated tumor cells engineered to secrete murine granulocyte-macrophage colonystimulating factor stimulates potent, specific, and long-lasting antitumor immunity. Proc Natl Acad Sci U S A. 1993;90:3539-3543.

25. van Elsas A, Hurwitz AA, Allison JP. Combination immunotherapy of B16 melanoma using anti-cytotoxic T lymphocyte-associated antigen 4 (CTLA-4) and granulocyte/macrophage colony-stimulating factor (GM-CSF)-producing vaccines induces rejection of subcutaneous and metastatic tumors accompanied by autoimmune depigmentation. J Exp Med. 1999;190(3):355-366.

26. Hurwitz AA, Yu TF, Leach DR, Allison JP. CTLA-4 blockade synergizes with tumor-derived granulocyte-macrophage colony-stimulating factor for treatment of an experimental mammary carcinoma. Proc Natl Acad Sci U S A. 1998;95(17):10067-10071.

27. Hurwitz AA, Foster BA, Kwon ED, et al. Combination immunotherapy of primary prostate cancer in a transgenic mouse model using CTLA-4 blockade. Cancer Res. 2000;60(9):2444-2448.

28. Gregor PD, Wolchok JD, Ferrone CR, et al. CTLA-4 blockade in combination with xenogeneic DNA vaccines enhances T-cell responses, tumor immunity and autoimmunity to self antigens in animal and cellular model systems. Vaccine. 2004;22(13-14):1700-1708.

29. Davila E, Kennedy R, Celis E. Generation of antitumor immunity by cytotoxic T lymphocyte epitope peptide vaccination, CpGoligodeoxynucleotide adjuvant, and CTLA-4 blockade. Cancer Res. 2003;63(12):3281-3288. 
30. Chambers CA, Sullivan TJ, Allison JP. Lymphoproliferation in CTLA4-deficient mice is mediated by costimulation-dependent activation of CD4+ T cells. Immunity. 1997;7(6):885-895.

31. Chambers CA, Sullivan TJ, Truong T, Allison JP. Secondary but not primary $\mathrm{T}$ cell responses are enhanced in CTLA-4-deficient CD8+ T cells. Eur J Immunol. 1998;28(10):3137-3143.

32. McCoy KD, Hermans IF, Fraser JH, Le Gros G, Ronchese F. Cytotoxic T lymphocyte-associated antigen 4 (CTLA-4) can regulate dendritic cell-induced activation and cytotoxicity of CD8(+) T cells independently of CD4(+) T cell help. J Exp Med. 1999;189(7): $1157-1162$.

33. Peggs KS, Quezada SA, Chambers CA, Korman AJ, Allison JP. Blockade of CTLA-4 on both effector and regulatory T cell compartments contributes to the antitumor activity of anti-CTLA-4 antibodies. J Exp Med. 2009;206(8):1717-1725.

34. Kaehler KC, Piel S, Livingstone E, Schilling B, Hauschild A, Schadendorf D. Update on immunologic therapy with anti-CTLA-4 antibodies in melanoma: identification of clinical and biological response patterns, immune-related adverse events, and their management. Semin Oncol. 2010;37(5):485-498.

35. Ribas A. Clinical development of the anti-CTLA-4 antibody tremelimumab. Semin Oncol. 2010;37(5):450-454.

36. Camacho LH, Antonia S, Sosman J, et al. Phase I/II trial of tremelimumab in patients with metastatic melanoma. J Clin Oncol. 2009;27(7):1075-1081.

37. Weber J. Ipilimumab: controversies in its development, utility and autoimmune adverse events. Cancer Immunol Immunother. 2009;58(5):823-830.

38. Attia P, Phan GQ, Maker AV, et al. Autoimmunity correlates with tumor regression in patients with metastatic melanoma treated with anti-cytotoxic T-lymphocyte antigen-4. J Clin Oncol. 2005;23(25):6043-6053.

39. Maker AV, Yang JC, Sherry RM, et al. Intrapatient dose escalation of anti-CTLA-4 antibody in patients with metastatic melanoma J Immunother. 2006;29(4):455-463.

40. Weber JS, O'Day S, Urba W, et al. Phase I/II study of ipilimumab for patients with metastatic melanoma. J Clin Oncol. 2008;26(36):5950-5956

41. Hodi FS, Butler M, Oble DA, et al. Immunologic and clinical effects of antibody blockade of cytotoxic T lymphocyte-associated antigen 4 in previously vaccinated cancer patients. Proc Natl Acad Sci U S A. 2008;105(8):3005-3010

42. Wolchok JD, Kluger $\mathrm{H}$, Callahan MK, et al. Nivolumab plus ipilimumab in advanced melanoma. N Engl J Med. 2013;369(2):122-133.

43. Topalian SL, Hodi FS, Brahmer JR, et al. Safety, activity, and immune correlates of anti-PD-1 antibody in cancer. $N$ Engl $J$ Med. 2012;366:2443-2454.

44. Wolchok JD, Neyns B, Linette G, et al. Ipilimumab monotherapy in patients with pretreated advanced melanoma: a randomised, double-blind, multicentre, phase 2, dose-ranging study. Lancet Oncol. 2010;11(2): $155-164$.

45. O’Day SJ, Maio M, Chiarion-Sileni V, et al. Efficacy and safety of ipilimumab monotherapy in patients with pretreated advanced melanoma: a multicenter single-arm phase II study. Ann Oncol. 2010;21(8):1712-1717.

46. Weber J, Thompson JA, Hamid O, et al. A randomized, double-blind, placebo-controlled, phase II study comparing the tolerability and efficacy of ipilimumab administered with or without prophylactic budesonide in patients with unresectable stage III or IV melanoma. Clin Cancer Res. 2009;15(17):5591-5598.

47. Korn EL, Liu PY, Lee SJ, et al. Meta-analysis of phase II cooperative group trials in metastatic stage IV melanoma to determine progressionfree and overall survival benchmarks for future phase II trials. $J$ Clin Oncol. 2008;26(4):527-534

48. Margolin K, Ernstoff MS, Hamid O, et al. Ipilimumab in patients with melanoma and brain metastases: an open-label, phase 2 trial. Lancet Oncol. 2012;13(5):459-465.
49. Di Giacomo AM, Ascierto PA, Pilla L, et al. Ipilimumab and fotemustine in patients with advanced melanoma (NIBIT-M1): an open-label, single-arm phase 2 trial. Lancet Oncol. 2012;13(9):879-886.

50. Hodi FS, O'Day SJ, McDermott DF, et al. Improved survival with ipilimumab in patients with metastatic melanoma. $N$ Engl $J$ Med. 2010;363(8):711-723.

51. Hoos A, Eggermont AM, Janetzki S, et al. Improved endpoints for cancer immunotherapy trials. J Natl Cancer Inst. 2010;102(18):1388-1397.

52. Robert C, Hodi FS, O'Day, et al. Re-induction with ipilimumab, gp100 peptide vaccine or a combination of both in a Phase III study of previously-treated patients with advanced melanoma: update of clinical characteristic of patients. In: 35th Congress of the European Society for Medical Oncology (ESMO); October 8-10, 2010; Milan, Italy.

53. Schwartzentruber DJ, Lawson DH, Richards JM, et al. gp100 peptide vaccine and interleukin-2 in patients with advanced melanoma. $N E n g l$ J Med. 2011;364(22):2119-2127.

54. Prieto PA, Yang JC, Sherry RM, et al. CTLA-4 blockade with ipilimumab: long-term follow-up of 177 patients with metastatic melanoma. Clin Cancer Res. 2012;18(7):2039-2047.

55. Robert C, Thomas L, Bondarenko I, et al. Ipilimumab plus dacarbazine for previously untreated metastatic melanoma. $N$ Engl $J$ Med. 2011;364(26):2517-2526.

56. Almubarak M, Abraham J. Research highlights: highlights from the latest articles in cancer immunotherapy. Immunotherapy. 2012;4(5):473-475.

57. ClinicalTrials.gov [homepage on the Internet]. National Institutes of Health. Available from: http://www.clinicaltrials.gov. Accessed July 23, 2013.

58. Ribas A, Chmielowski B, Glaspy JA. Do we need a different set of response assessment criteria for tumor immunotherapy? Clin Cancer Res. 2009;15(23):7116-7118.

59. Ribas A, Comin-Anduix B, Economou JS, et al. Intratumoral immune cell infiltrates, FoxP3, and indoleamine 2,3-dioxygenase in patients with melanoma undergoing CTLA4 blockade. Clin Cancer Res. 2009; 15:390-399.

60. Wolchok JD, Hoos A, O'Day S, et al. Guidelines for the evaluation of immune therapy activity in solid tumors: immune-related response criteria. Clin Cancer Res. 2009;15:7412-7420.

61. van Elsas A, Sutmuller RP, Hurwitz AA, et al. Elucidating the autoimmune and antitumor effector mechanisms of a treatment based on cytotoxic T lymphocyte antigen-4 blockade in combination with a B16 melanoma vaccine: comparison of prophylaxis and therapy. $J$ Exp Med. 2001;194(4):481-489.

62. Maker AV, Phan GQ, Attia P, et al. Tumor regression and autoimmunity in patients treated with cytotoxic $\mathrm{T}$ lymphocyte-associated antigen 4 blockade and interleukin 2: a phase I/II study. Ann Surg Oncol. 2005;12(12):1005-1016.

63. Downey SG, Klapper JA, Smith FO, et al. Prognostic factors related to clinical response in patients with metastatic melanoma treated by CTL-associated antigen-4 blockade. Clin Cancer Res. 2007;13(22 Pt 1): 6681-6688.

64. Ku GY, Yuan J, Page DB, et al. Single-institution experience with ipilimumab in advanced melanoma patients in the compassionate use setting: lymphocyte count after 2 doses correlates with survival. Cancer. 2010;116(7):1767-1775.

65. Yang A, Kendle RF, Ginsberg BA, et al. CTLA-4 blockade with ipilimumab increases peripheral CD8+ cells: correlation with clinical outcomes. J Clin Oncol. 2010;28:15s(suppl; abstract 2555).

66. Marshall MA, Ribas A, Huang B. Evaluation of baseline serum C-reactive protein (CRP) and benefit from tremelimumab compared to chemotherapy in first-line melanoma. J Clin Oncol. 2010;28:15s(suppl; abstract 2609).

67. Hamid O, Schmidt H, Nissan A, et al. A prospective phase II trial exploring the association between tumor microenvironment biomarkers and clinical activity of ipilimumab in advanced melanoma. $J$ Transl Med. 2011;9:204. 
68. Cetindere T, Nambiar S, Santourlidis S, Essmann F, Hassan M. Induction of indoleamine 2, 3-dioxygenase by death receptor activation contributes to apoptosis of melanoma cells via mitochondrial damage-dependent ROS accumulation. Cell Signal. 2010;22(2):197-211.

69. Oble DA, Loewe R, Yu P, Mihm MC Jr. Focus on TILs: prognostic significance of tumor infiltrating lymphocytes in human melanoma. Cancer Immun. 2009;9:3.

70. Liakou CI, Kamat A, Tang DN, et al. CTLA-4 blockade increases IFN-gamma-producing CD4+ICOShi cells to shift the ratio of effector to regulatory T cells in cancer patients. Proc Natl Acad Sci U S A. 2008;105:14987-14992.
71. Carthon BC, Wolchok JD, Yuan J, et al. Preoperative CTLA-4 blockade: tolerability and immune monitoring in the setting of a presurgical clinical trial. Clin Cancer Res. 2010;16(10):2861-2671.

72. Fu T, He Q, Sharma P. The ICOS/ICOSL pathway is required for optimal antitumor responses mediated by anti-CTLA-4 therapy. Cancer Res. 2011;71(16):5445-5454.

73. Verschraegen $\mathrm{C}$. The monoclonal antibody to cytotoxic T lymphocyte antigen 4, ipilimumab, in the treatment of melanoma. Cancer Manag Res. 2012;4:1-8.

\section{Publish your work in this journal}

Clinical, Cosmetic and Investigational Dermatology is an international, peer-reviewed, open access, online journal that focuses on the latest clinical and experimental research in all aspects of skin disease and cosmetic interventions. All areas of dermatology will be covered; contributions will be welcomed from all clinicians and basic science researchers globally. This journal is indexed on CAS The manuscript management system is completely online and includes a very quick and fair peer-review system, which is all easy to use. Visit http://www.dovepress.com/testimonials.php to read real quotes from published authors.

Submit your manuscript here: http://www.dovepress.com/clinical-cosmetic-and-investigational-dermatology-journal 